\title{
Clinical Characteristics of Cerebrovascular Pathology with Patients Suffering from Ph-Negative Myeloproliferative Disease
}

\author{
Marine M. Tanashyan ${ }^{a}$ Polina I. Kuznetsova ${ }^{a} \quad$ Alla A. Shabalina ${ }^{b}$ \\ Anton A. Raskurazhev ${ }^{a}$ Olga V. Lagoda ${ }^{a}$ Irina N. Subortseva ${ }^{c}$ \\ Anait L. Melikyanc \\ ${ }^{a}$ Angioneurology and b ${ }^{\mathrm{L}}$ aboratory Diagnostics, Research Center of Neurology, and \\ 'Research Center of Hematology, Moscow, Russia
}

\section{Key Words}

Ph-negative myeloproliferative disorder $\cdot$ Cerebrovascular disease $\cdot$ Diagnosis

\begin{abstract}
Background: Disturbances of microcirculation play a significant role in the development and progression of both acute and chronic cerebrovascular diseases (CVD) and may be associated with different hemogram abnormalities. One of the reasons of the prothrombogenic state of the endothelium is the increase in the number of blood corpuscles leading to (non-Ph) myeloproliferative disorders (MPD) including essential thrombocythemia (ET), polycythemia vera (PV), and primary myelofibrosis (PM). Materials and Methods: The study included 167 patients: 102 patients with Ph-MPD and the control group comprising 65 patients with CVD. According to MPD subtype, the patients were divided into three groups: patients with ET (37\%, $\mathrm{n}=38$, male/female 7/31, age $52 \pm 7$ years), those with PV (40\%, $n=41$, male/female 20/21, age $50 \pm 6$ years) and those with PM (23\%, $\mathrm{n}=23$, male/female 5/18, age $54 \pm 4$ years). $\boldsymbol{R e}$ sults: In $79 \%(n=81)$ of cases in the study group (with Ph-MPD), patients had chronic CVD, with the most frequently identified symptoms being asthenia (92\%) and headache (72\%). Headache in Ph-MPD patients was more frequently (86\%) associated with PM, while in patients with PV and ET it was equally distributed (70\%). Neurological symptoms in $53 \%$ of cases were associated with focal changes of the brain on MRI localized in the subcortical area of the frontal and parietal lobes. Twenty-one (21\%) patients suffered an acute cerebrovascular accident, 8 of them had thrombotic occlusion of one of the internal carotid arteries leading to hemispheric infarcts. Endothelial function (as measured by flow-dependent dilation of the brachial artery) was severely impaired in all study groups (median 5\% with normal cut-off at $10 \%$ ), the lowest degree of vasodilator activity being specific for patients with a history of
\end{abstract}


stroke $(p=0.011)$. Conclusion: Patients suffering from MPD had asymptomatic focal changes in the brain in the absence of concomitant vascular disease (hypertension, atherosclerotic vascular disease, heart rhythm disorders) in 50\% of cases. MPD, while remaining un- or underdiagnosed, presents a major concern in the cerebrovascular setting. A large number of thrombotic strokes occurring in patients with ET underline the necessity of early diagnostics and preventive therapy in these patients.

(C) 2016 The Author(s)

Published by S. Karger AG, Basel

\section{Introduction}

Cerebrovascular disease (CVD) is a global problem of modern healthcare leading to high mortality rates. The percentage of mortality due to acute and chronic CVD is $11.13 \%$ [1], which makes it the second cause of death in the world [2]. High rates of disability, mortality and economic burden caused by CVD underline the necessity of broad research into various aspects of the etiology, clinical presentation and progression of vascular brain pathology.

Both acute and chronic CVD are predominantly accompanied by rheological abnormalities, the latter occurring in a number of cases due to an increase in blood corpuscles. One of the reasons of elevated erythrocyte and/or platelet count is Ph-negative myeloproliferative neoplasms, i.e. excessive proliferation of one or more cell lines of myelopoiesis [3].

High rate of hematocrit significantly increases the risk of thrombotic complications in arterial and venous vessels. Thrombosis of the carotid and vertebrobasilar region with hemispheric infarction is a life-threatening condition. Mortality rates in patients with $\mathrm{Ph}$-negative myeloproliferative disorder (Ph-MPD) are 1.5 times higher than in the general population; hence, it is becoming increasingly important to elucidate the mechanisms of large- and smallvessel thrombotic events $[4,5]$. The present study aims at identifying clinical and laboratory features of CVD in the setting of Ph-MPD.

\section{Methods}

The study included 167 patients: 102 patients with Ph-MPD and the control group comprising 65 patients with CVD. According to MPD subtype, the patients were divided into three groups (WHO 2008): patients with essential thrombocythemia (ET) (37\%, $n=38$, male/ female $7 / 31$, age $52 \pm 7$ years), those with polycythemia vera (PV) $(40 \%, n=41$, male/female $20 / 21$, age $50 \pm 6$ years) and those with primary myelofibrosis (PM) $(23 \%, n=23$, male/ female $5 / 18$, age $54 \pm 4$ years).

Patients with advanced stages of cardiac disease, renal and/or liver failure, hypertension or carotid atherosclerosis were excluded from the study group. The control group included patients with CVD and concomitant cardiovascular pathology. Age $>58$ years was an exclusion criterion for all groups.

All patients underwent a thorough clinical and neurological exam: MRI scan of the brain, MR angiography, MR imaging of the cerebral venous sinuses; duplex sonography of the brachiocephalic arteries with the measurement of flow-dependent dilation of the brachial artery (as an endothelial dysfunction test with a normal cut-off of $>10 \%$ ); complex blood tests (including hemorheology, hemostasis, complete blood count, platelet and erythrocyte aggregation, morphofunctional properties of red blood cells), and a specific biochemical tourniquet test (applied for endothelial dysfunction estimation). 


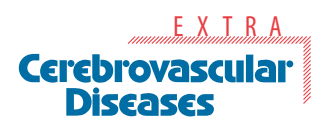

\begin{tabular}{l|l}
\hline Cerebrovasc Dis Extra 2016;6:66-70 \\
\hline DOI: 10.1159/000448597 & $\begin{array}{l}\text { (c) 2016 The Author(s). Published by S. Karger AG, Basel } \\
\text { www.karger.com/cee }\end{array}$ \\
\hline
\end{tabular}

Tanashyan et al:: Clinical Characteristics of Cerebrovascular Pathology with Patients Suffering from Ph-Negative Myeloproliferative Disease
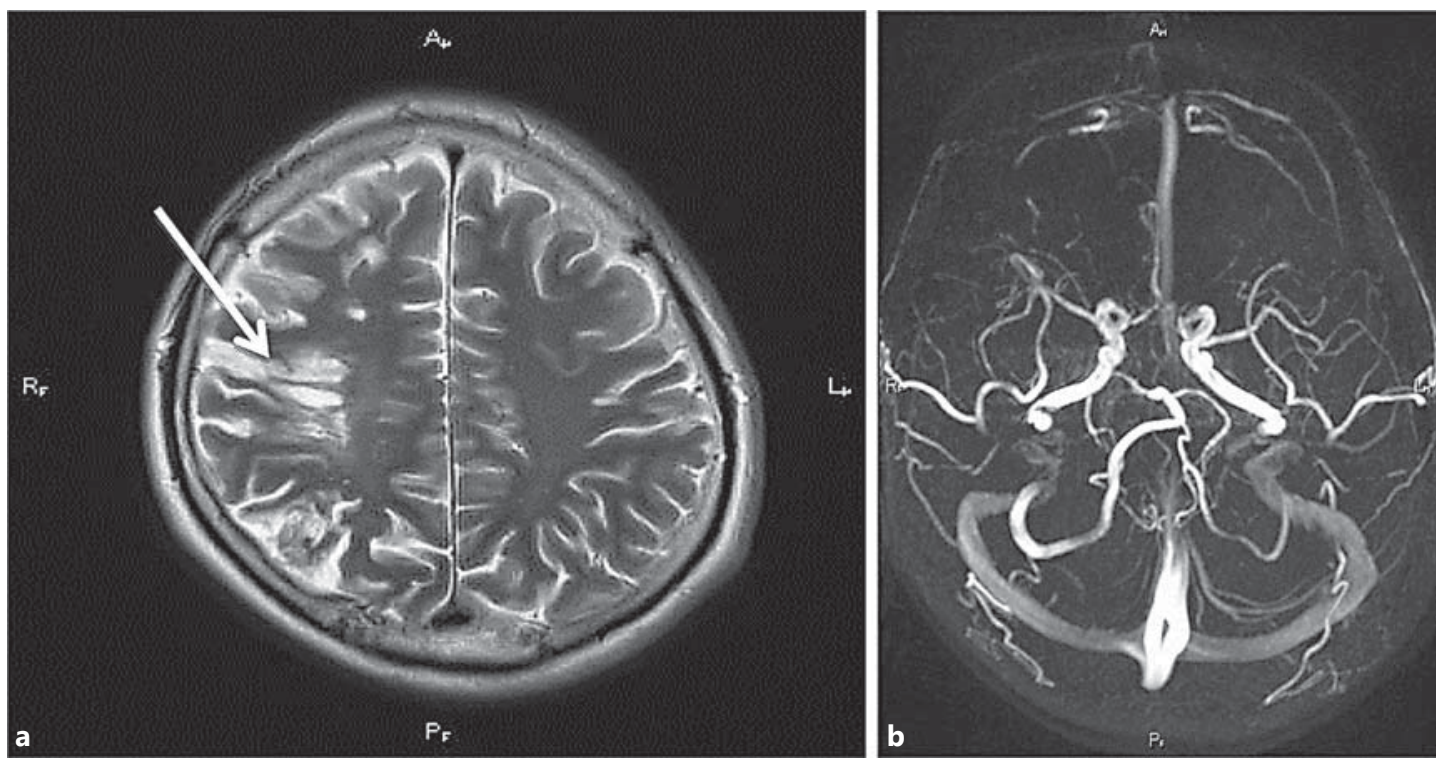

Fig. 1. a Patient R., 52 years. Massive right-hemispheric infarction due to thrombotic occlusion of the ICA (first $24 \mathrm{~h}$ ). b Same patient. Blood flow recovery in the right middle cerebral artery (MR angiography, $48 \mathrm{~h}$ after stroke).

\section{Results}

In $79 \%(n=81)$ of cases in the study group (with Ph-MPD), patients had chronic CVD, with the most frequently identified symptoms being asthenia (92\%) and headache $(72 \%)$. Other manifestations such as vestibular disorders were observed in $14 \%$ patients $(n=11)$, motor disorders in 5\% $(n=4)$ and sensory disorders in 17\% $(n=14)$ of cases. Headache in $\mathrm{Ph}-\mathrm{MPD}$ patients was more frequently (86\%) associated with PM, while in patients with PV and ET it was equally distributed (70\%). The main clinical features of headache in patients with Ph-MPD were as follows: constant, of moderate intensity, often associated with photopsia. Neurological symptoms in $53 \%$ of cases with chronic CVD $(n=43)$ were associated with focal changes of the brain on MRI localized in the subcortical area of the frontal and parietal lobes. Of note, no lesions were observed in the deep subcortical white matter - this may as well be attributed to our specific exclusion of patients with hypertension, heart rhythm dysfunction and carotid atherosclerosis.

Twenty-one (21\%) patients suffered an acute cerebrovascular accident, 8 of them had thrombotic occlusion of one of the internal carotid arteries (ICA) leading to hemispheric infarcts. We observed a male patient with ET aged 52 years who had a right-hemispheric infarction due to a thrombotic occlusion of the unilateral ICA (fig. 1a). After $48 \mathrm{~h}$, the same patient had a recovery of blood flow in the right middle cerebral artery, as shown on the MR angiography (fig. 1b).

Thirteen patients had small cortical infarctions, possibly related to hemorheological micro-occlusion. Endothelial function (as measured by ultrasound) was severely impaired in all study groups, the lowest degree of vasodilator activity being specific for patients with a history of stroke ( $\mathrm{p}=0.011)$.

Hemorheologic and hemostasis abnormalities were not statistically significantly changed between subtypes of Ph-MPD, which allowed to view them as a whole group. The studied 

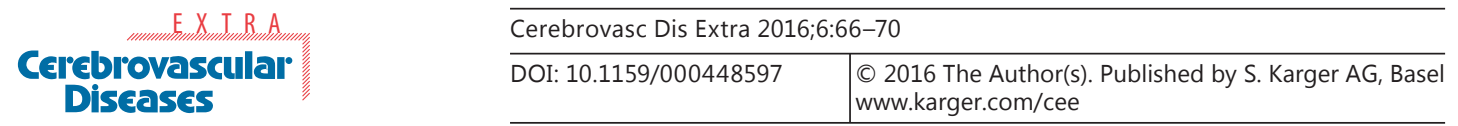

Tanashyan et al.: Clinical Characteristics of Cerebrovascular Pathology with Patient Suffering from Ph-Negative Myeloproliferative Disease

Table 1. Hemorheology and hemostasis parameters in CVD patients with or without Ph-MPD

\begin{tabular}{|c|c|c|c|c|c|}
\hline & $\begin{array}{l}\text { Stroke } \\
\text { (with MPD) }\end{array}$ & $\begin{array}{l}\text { Stroke } \\
\text { (without MPD) }\end{array}$ & $\begin{array}{l}\text { Chronic CVD } \\
\text { (with MPD) }\end{array}$ & $\begin{array}{l}\text { Chronic CVD } \\
\text { (without MPD) }\end{array}$ & $\begin{array}{l}\text { Reference } \\
\text { values }\end{array}$ \\
\hline ADP-PA & $\begin{array}{l}28.2 \pm 3.01^{*} \\
p=0.001\end{array}$ & $38.3 \pm 4.3$ & $29.5 \pm 3.1$ & $41.4 \pm 4.5$ & $40-46$ \\
\hline Adr-PA & $29.5 \pm 3.2$ & $35.4 \pm 4.6$ & $25.01 \pm 2.8$ & $36.8 \pm 4.2$ & $37-43$ \\
\hline RBC deformity & $\begin{array}{l}0.43 \pm 0.037^{*} \\
p=0.0012\end{array}$ & $\begin{array}{l}0.51 \pm 0.043^{* *} \\
\mathrm{p}=0.013\end{array}$ & $\begin{array}{l}0.45 \pm 0.044^{* *} \\
\mathrm{p}=0.022\end{array}$ & $0.53 \pm 0.023$ & $0.52-0.55$ \\
\hline Aggregate stability, s & $\begin{array}{l}267.9 \pm 18.8^{*} \\
p=0.0031\end{array}$ & $185 \pm 20.6$ & $210.5 \pm 16.7$ & $125.6 \pm 14.5$ & $79-110$ \\
\hline von Willebrand factor & $111 \pm 9.3$ & $98.6 \pm 10.5$ & $117.5 \pm 12.8$ & $104.2 \pm 10.6$ & $79-110$ \\
\hline Hematocrit & $\begin{array}{l}50 \pm 2.27^{*} \\
p=0.0062\end{array}$ & $44.6 \pm 2.81$ & $46.4 \pm 2.9$ & $39 \pm 2.6$ & $35-45$ \\
\hline Antithrombin III & $95.6 \pm 8.27$ & $110.4 \pm 10.9$ & $112.5 \pm 13.2$ & $105.4 \pm 10.9$ & $71-115$ \\
\hline Fibrinolytic activity & $\begin{array}{l}12.7 \pm 0.11^{*} \\
p=0.018\end{array}$ & $17.2 \pm 0.12$ & $\begin{array}{l}13.3 \pm 0.11^{* *} \\
p=0.016\end{array}$ & $18.6 \pm 0.14$ & $14-18$ \\
\hline Fibrinolysis index & $\begin{array}{l}0.60 \pm 0.05^{*} \\
p=0.022\end{array}$ & $0.9 \pm 0.012$ & $\begin{array}{l}0.58 \pm 0.01^{* *} \\
p=0.036\end{array}$ & $1.0 \pm 0.05$ & $0.8-1.2$ \\
\hline
\end{tabular}

ADP-PA = Adenosine diphosphate-induced platelet aggregation; Adr-PA = adrenaline-induced platelet aggregation; $\mathrm{RBC}=$ red blood cell. * Statistically significant differences between groups, $\mathrm{p}<0.05$ (stroke with MPD vs. reference). ${ }^{* *}$ Study versus control group.

blood parameters compared to controls (MPD vs. chronic and acute CVD) are presented in table 1.

Erythrocyte deformability characteristics in patients with CVD and MPD differ significantly not only from reference values, but from patients in the control group (CVD without MPD). There were no statistically significant differences between levels of von Willebrand factor and antithrombin III in patients of both study and control groups. Hematocrit levels were higher in patients with Ph-MPD, while fibrinolytic activity did not differ between groups (though it was lower than the reference values).

Endothelial dysfunction rates were lower in patients with Ph-MPD compared to CVD patients without this pathology. Athrombogenic potential is decreased predominantly due to the antiaggregational and fibrinolytical components, while the anticoagulational function of the endothelium remained relatively intact.

\section{Discussion}

CVD may present as an initial manifestation of Ph-MPD [6]. Chronic brain ischemia often manifests itself with headache and/or asthenia [7]. There exists a firm link between hematological characteristics and cephalgia, supported by several studies $[8,9]$ where it has been demonstrated that in PV and ET, headache is often a first symptom of Ph-MPD [10]. Our findings confirm these data, with as much as $70 \%$ of patients referring to their physician with headache as the main complaint (which preceded the diagnosis of Ph-MPD). Headache presenting as a first and foremost neurological complaint was most frequently of a secondary origin and was closely connected to the degree of platelet count. Patients with Ph-MPD present with focal changes in the subcortical white matter in the frontal and parietal lobes. We suggest that this may be due to the vascular architecture of the terminal branches of the middle 
cerebral artery, which, descending from the convex, are more prone to thrombosis. Endothelial dysfunction increases the risk of thrombosis in the microcirculatory setting as well as in large vessels.

\section{Conclusion}

(1) Headache (predominantly of a secondary origin) is a major clinical presentation of MPD, often being one of the earliest neurological symptoms. (2) MRI changes include corticalsubcortical lesions of vascular origin often not presenting clinically 'silent' brain lesions. (3) The lowest endothelial dysfunction rates were observed in patients with stroke, which confirms the decrease of the functional compensating capacity of the vascular endothelium leading to acute cerebrovascular events. (4) MPD, while remaining un- or underdiagnosed, presents a major concern in the cerebrovascular setting.

\section{References}

1 Institute for Health Metrics and Evaluation: GBD Compare: Global Burden of Disease data visualizations. Seattle, University of Washington, 2010. http://vizhub.healthdata.org/gbd-compare/ (accessed July 31, 2014).

2 Mozaffarian D, Benjamin EJ, Go AS, et al: Heart disease and stroke statistics - 2015 update: a report from the American Heart Association. Circulation 2015;131:e29-e322.

7 Tefferi A: Pathogenesis of myelofibrosis with myeloid metaplasia. J Clin Oncol 2005;23:8520-8530.

4 Brodmann S, Passweg JR, Gratwohl A, Tichelli A, Skoda RC: Myeloproliferative disorders: complications, survival and causes of death. Ann Hematol 2000;79:312-318.

5 De Stefano V, Za T, Rossi E, et al: Influence of the JAK2 V617F mutation and inherited thrombophilia on the thrombotic risk among patients with essential thrombocythemia. Haematologica 2009;94:733-737.

6 Blumenthal DT, Glenn MJ: Neurological manifestation of hematological disorders. Neurol Clin 2002;20:265281, viii.

7 Michiels JJ, Berneman Z, Schroyens W, et al: Platelet-mediated erythromelalgic, cerebral, ocular and coronary microvascular ischemic and thrombotic manifestations in patients with essential thrombocythemia and polycythemia vera: a distinct aspirin-responsive and coumadin-resistant arterial thrombophilia. Platelets 2006; 17:528-544.

8 Ferrant A: What clinical and laboratory data are indicative of polycythemia and when are blood volume samples needed? Nouv Rev Fr Hematol 1994;36:151-154.

19 Newton LK: Neurologic complications of polycythemia and their impact on therapy. Oncology 1990;4:59-64.

10 Frewin R, Dowson A: Headache in essential thrombocythaemia. Int J Clin Pract 2012;66:976-983. 